\title{
Self-assembled quantum dots: The route to novel optical, electronic, magnetic and superconducting properties
}

\author{
S BANDYOPADHYAY \\ Department of Electrical Engineering, University of Nebraska, Lincoln, Nebraska 68588-0511, USA
}

\begin{abstract}
We report recent results pertaining to the magnetic, optical, electronic, superconducting, and topographic properties of electrochemically self-assembled quantum dots. These dots self-order into twodimensional hexagonal-close-packed arrays that are among the most periodic reported so far. They have revealed interesting properties with potential applications in magnetics, electronics, non-linear optics and novel neural architectures for ultrafast computation and signal processing.
\end{abstract}

Keywords. Self-assembly; quantum dots; non-linear optics; nanomagnetism; electronic-memory; quantumarchitectures.

\section{Introduction}

There is significant current interest in synthesizing quantum dots by self-assembly. A number of techniques have been demonstrated and have produced quantum dot arrays with varying degrees of spatial ordering. StranskiKrastanow (S-K) growth of quantum dots by coherent islanding (Bimberg et al 1998) generally does not result in any spatial ordering. On the other hand, chemical self-assembly of metal clusters (Murray et al 1995; Andres et al 1996) - linked by polymers and organic molecules-leads to excellent ordering but is limited in the choice of materials. Disordered or poorly ordered dots may have attractive optical properties and might lead to useful quantum dot lasers, but their applications in transport, magnetics and electronics are limited. On the other hand, clusters are often too small for many optical (or even electronic) devices since their properties can be dominated by surface states that are difficult or impossible to engineer.

Recently, two self-assembly techniques have been reported that result in exceptionally ordered twodimensional arrays of quantum dots (Bandyopadhyay et al 1996; Miller and Bandyopadhyay 1998) that are large enough to be useful as electronic or optical devices. We will briefly describe these techniques and refer the reader to Bandyopadhyay et al (1996) and Miller and Bandyopadhyay (1998) for details. A quantitative theory describing the underlying phenomena responsible for the unprecedented degree of ordering has been developed and described by Yuzhakov et al (1997).

\section{Electrochemical self-assembly}

We will describe two electrochemical techniques, based on the electropolishing and anodization of aluminum, that result in highly ordered arrays of quantum dots and wires. Both these techniques are simple, inexpensive, gentle and result in fast throughput.

\subsection{Electropolishing of aluminum}

The first technique involves electropolishing a $99.99 \%$ pure $\mathrm{Al}$ foil (>100 $\mu \mathrm{m}$ in thickness). The foil is degreased ultrasonically in dichloromethane solution for $5 \mathrm{~min}$, followed by thorough rinsing in distilled water. It is then electropolished in $\mathrm{L} 1$ electrolyte consisting of $62 \mathrm{cc}$ perchloric acid, $700 \mathrm{cc}$ ethanol, $100 \mathrm{cc}$ butyl cellusolve and $137 \mathrm{cc}$ distilled water. The electropolishing is carried out at various voltages for various durations. If the electropolishing voltage is $50 \mathrm{~V}$ and the duration $10 \mathrm{sec}$, one obtains a highly periodic array of ridges on the surface of aluminum. On the other hand, if the electropolishing voltage is $60 \mathrm{~V}$ and the duration $30 \mathrm{sec}$, one obtains a hexagonal closed packed array of conical hills resembling an 'egg-carton'.

Raw atomic force micrographs of the strip and eggcarton' pattern are shown in figure 1. Instead of using a foil to produce these patterns, one could also electroplate a layer of $\mathrm{Al}$ on a chosen substrate and then produce the same surface features by electropolishing. A mild etch with dilute bromine/methanol can subsequently remove the troughs and leave behind the crests to form isolated strips or islands of $\mathrm{Al}$ on the substrate. These could be used as natural masks to mesa isolate quantum wires or dots in the underlying substrate using reactive ion etching. Etching of $9 \mathrm{~nm}$ pillars with gold islands as a natural mask have been demonstrated in the past (Tada et al 1998) and lends credence to such an approach.

The electropolished patterns have been studied extensively by atomic force microscopy (Ricker et al 1996). The standard deviation in the diameter of the hills- 
measured as full width at half maximum-is less than $1 \%$. These are among the most ordered and size controlled self-assembled arrays of quantum dots and wires reported to date.

\subsection{Anodization of aluminum}

Another technique for producing quasi ordered twodimensional arrays of quantum dots involves anodization of aluminum. When an electropolished aluminum foil is anodized in an appropriate acid, a thin nanoporous film of alumina forms on the surface. The pores in the alumina film spontaneously self-order into a twodimensional quasi-periodic array. The pore size depends on the acid chosen. Typically, the pore diameter is about $100 \mathrm{~nm}$ for phosphoric or oxalic acid, and $10 \mathrm{~nm}$ for sulfuric acid. The standard deviation in the pore diameter can be well controlled to within $10 \%$.
A variant of this technology has been known in the colouring industry for at least the last fifty years. Only recently it has attracted attention in the context of nanotechnology and quantum structures (Diggle et al 1969; Moskovitz 1993; Huber et al 1994; Martin 1994; Routkevich et al 1996).

2.2a Self-ordering of the pores: There have been two new advancements which improve the regimentation of the pore array. In one technique ( $\mathrm{Li}$ 1997), anodization is carried out for several hours to create a thick porous alumina film. This film is then stripped and anodization is repeated. Several repetitions leave behind ordered arrays of scallops on the bare aluminum surface which act as nucleation sites for pores in the next round of anodization. The chemistry of this process is not well understood, but repeated anodization certainly improves the ordering.

\section{TWO TYPICAL ELECTROPOLISHED SURFACE STRUCTURES}
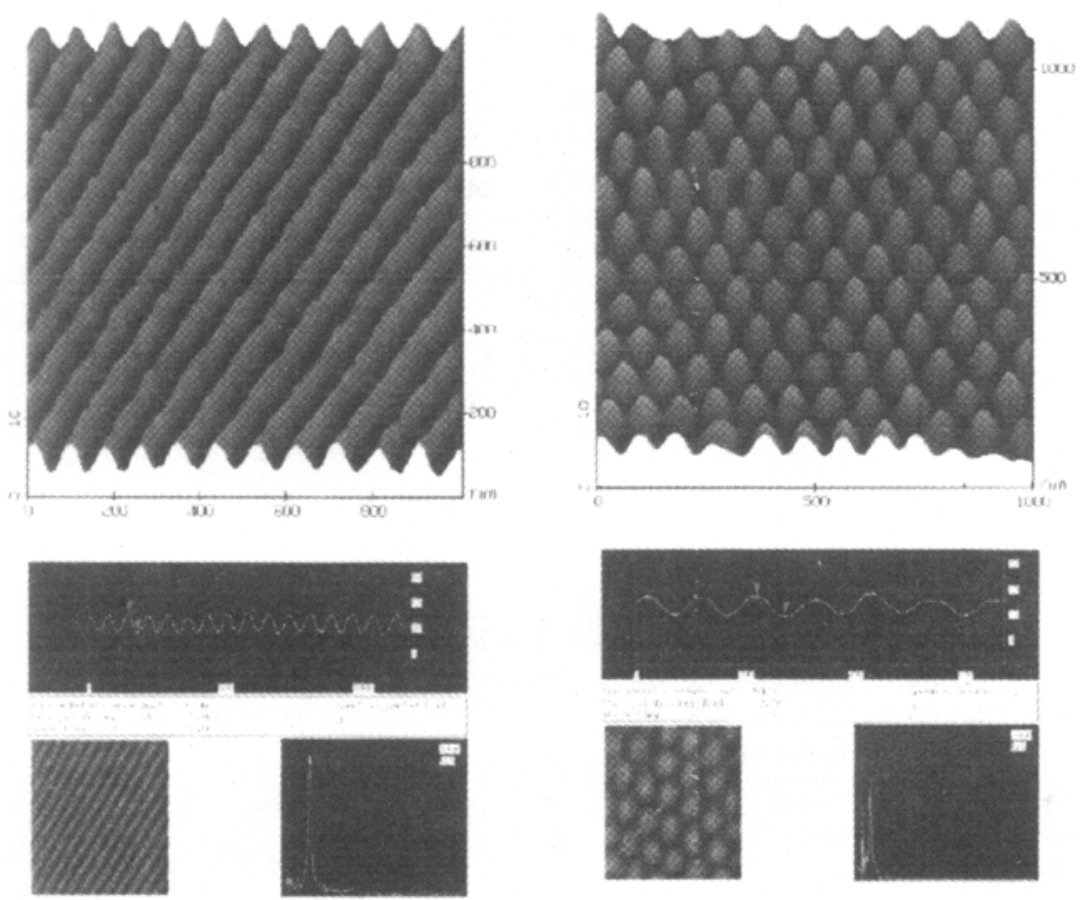

Figure 1. Surface features produced on a thin aluminum film by electropolishing the film in a solution of perchloric acid, butyl cellusolve, ethanol and distilled water. The stripe pattern is formed when the electropolishing duration is $10 \mathrm{sec}$ and the voltage is $50 \mathrm{~V}$, while the 'egg-carton' pattern is formed when the electropolishing duration is $30 \mathrm{sec}$ and the voltage is $60 \mathrm{~V}$. The stripes can be used for producing quantum wires and the 'egg-carton' pattern can be used as a self-assembled mask to create quantum dots with auto-registered contacts. The areas scanned here are $1 \mu \mathrm{m} \times 1 \mu \mathrm{m}$. The lower panels show the depth profiles and Fourier transforms of the depth profiles along arbitrary lines on the surface. The well-resolved Fourier peaks attest to the high degree of ordering and periodicity. 
In the second technique (Masuda et al 1997), a SiC mold containing a highly periodic array of hills - much like the 'egg-carton' in figure 1-is produced by electron beam lithography and reactive ion etching. This mold is then pressed on to the bare aluminum film to indent the surface. When the indented $\mathrm{Al}$ film is anodized, the pores nucleate selectively at the indentations. Highly ordered two-dimensional arrays of pores have been formed by this technique.

\subsection{Pore filling and formation of quantum dot arrays}

The pores in the alumina film can be filled with the material of choice by ac electrodeposition. For instance, if we want to fill the pores with cobalt, the porous film is first washed in acid to 'round' the pores and remove debris, then washed in distilled and deionized water. The film is then placed in a non-cyanide bath that does not attack alumina and ac current is passed at 15-20 V rms (frequency $100-200 \mathrm{~Hz}$ ) using the aluminum substrate and a graphite rod immersed in the bath as electrodes. The bath contains an aqueous solution of $\mathrm{CoSO}_{4}$. The $\mathrm{Co}^{++}$preferentially deposits within the pores which offer the least impedance path between the two electrodes. The degree of pore filling depends on the duration of electrodeposition. Typically, a $5 \mathrm{sec}$ deposition would result in filling the pores to a height of $10 \mathrm{~nm}$; while a $20 \mathrm{sec}$ deposition can fill a pore up to $200 \mathrm{~nm}$ (Bandyopadhyay et al 1996; Miller and Bandyopadhyay 1998). Successful pore filling by this process has been verified by cross-sectional transmission electron microscopy (Bandyopadhyay et al 1996; Yue et al 1996;

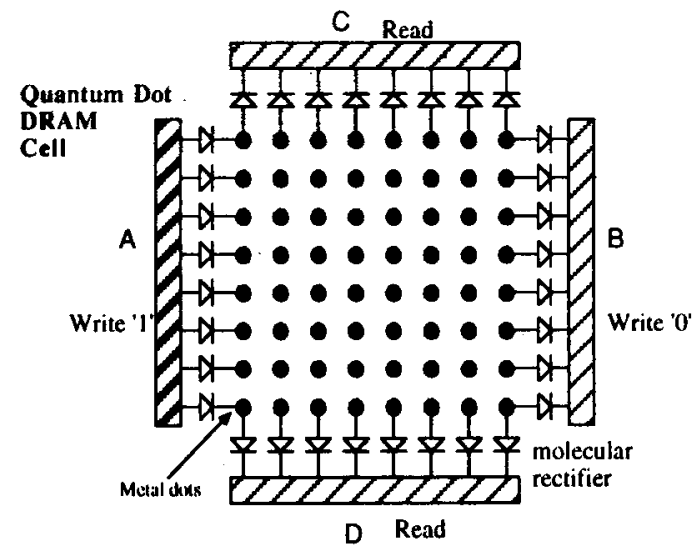

Figure 2. Schematic of a self-assembled quantum-dot memory. The dots are the storage elements and the metal lines are used to reading and writing data. The dots are all connected to each other by conjugated molecular wires that are not shown for the sake of clarity. The diodes are molecular rectifiers. The entire cell can be self-assembled except the metal lines which may be delineated by $\mathrm{X}$-ray lithography.
Miller and Bandyopadhyay 1998) and variable angle spectroscopic ellipsometry.

In order to fill the pores with a compound like CdS, the alumina film is first ac electrolysed in sulfuric acid for $10 \mathrm{sec}$ with an rms current density of $40 \mathrm{~mA} / \mathrm{cm}^{2}$ which leaves behind $\mathrm{S}^{--}$ions in the pores. This is followed by immersing the film in boiling deionized water containing $10 \% \mathrm{CdSO}_{4}$. The cation $\mathrm{Cd}^{++}$reacts with the $\mathrm{S}^{--}$adsorbed in the pore walls to form CdS.

The materials electrodeposited in the pores are usually polycrystalline. The impregnated films are annealed at a suitable temperature to improve crystallanity. The end product is an array of cylindrical quantum dots (or 'dashes') dispersed in a quasi-periodic two-dimensional hexagonal close packed arrangement in an alumina matrix.

Quantum dots of various materials formed by this technique have been extensively characterized with energy-dispersive analysis of X-ray, Auger spectroscopy, ESCA, photoluminescence, Raman spectroscopy, absorption spectroscopy, microwave surface resistance measurements, ellipsometry, magnetotransport experiments, non-degenerate pump-probe spectroscopy, currentvoltage measurements, etc (Bandyopadhyay et al 1996; Yue et al 1996; Miller and Bandyopadhyay 1998). From the optically measured blue-shift of the band-edge of CdS dots deposited in $10 \mathrm{~nm}$ pores, we have estimated that the effective diameter of the $\mathrm{CdS}$ dots is only about $3.5 \mathrm{~nm}$, even though the physical diameter measured by TEM is $10 \mathrm{~nm}$. We speculate that interface states deplete the boundary of the quantum dots leaving behind a central core of $3.5 \mathrm{~nm}$ in diameter which is optically active in optical experiments.

\section{Applications}

\subsection{Electronic circuit applications}

Quantum dots self-assembled by the first approach (electropolishing) may have a number of fascinating applications. One intriguing application involves the synthesis of powerful neural architecture chips for computation and signal processing. This application has been widely studied and a number of papers have appeared in the literature covering this topic (Roychowdhury et al 1996, 1997).

Another potential application is in ultrafast and lowpower dynamic random access memory (DRAMs).

A schematic for a quantum-dot DRAM cell is shown in figure 2. Addressing lines (or bit/word lines) to address a specific cell are not shown for the sake of clarity.

The memory works as follows: Line $\mathrm{A}$ is used for writing a ' 1 ' and B for writing a ' 0 '. Lines $C$ and $D$ are for reading. Line $\mathrm{A}$ is normally held low and all other lines are normally held high. To write a ' 1 ', A 
is raised high to forward bias the diodes connected to it. This charges the metal dots to a high voltage state which is interpreted as a stored ' 1 ' just as in a single capacitor DRAM. In the next clock cycle, A is lowered. The diodes connected to line $\mathrm{A}$ are reverse biased while all other diodes have zero- or slight reverse-bias. Thus, the dots cannot discharge and a ' 1 ' is stored. To write a ' 0 ', line $B$ is lowered to ground and the dots discharge through the diodes connected to $\mathrm{B}$. Line $\mathrm{A}$ is already low and diodes connected to lines $C$ and $D$ (which are held high) are reverse biased. Thus the clusters remain at the low voltage state and a ' 0 ' is stored.

To read stored data, lines $\mathrm{C}$ and $\mathrm{D}$ are lowered to ground. If the stored bit was a ' 1 ', the lines discharge and current flows in lines $\mathrm{C}$ and $\mathrm{D}$. If the stored bit was a 0 , no current flows.

All read/write operations are performed at the edges of the cell and all connections except those to the bit/word lines are self-assembled. Successive layers of cells may be vertically stacked to realize 3-D memory with bit density exceeding $10^{12} / \mathrm{cm}^{3}$.

Note that even one metal dot (instead of many as shown in the figure) could in principle act as a DRAM cell and store a binary data bit. This would increase the bit density, but is not advised. The use of many $(\sim 100)$ dots to represent a single stored bit introduces redundancy and consequently immense fault- and defect-tolerance. Similarly, the existence of two read lines instead of one, guarantees some fault tolerance in reading the stored bit.

To synthesize this memory, we proceed as follows. First, a $100 \mu \mathrm{m}$ thick layer of aluminum is electroplated on a chosen substrate. It is then electropolished to create the 'egg-carton' pattern and etched in dilute bromine/ methanol to selectively remove the troughs. This results in a periodic array of $\mathrm{Al}$ islands on the surface.

Cell areas are then delineated by $\mathrm{X}$-ray lithography which is not a direct write method and hence has a fast throughput. A set of metal lines (A, B, C and D) are then evaporated through a mask around each cell area. $\mathrm{A}$ very thin layer of $\mathrm{Au}$ is then evaporated on the surface. If the thickness is well below the percolation limit, the Au will form isolated islands or clusters of about $1-10 \mathrm{~nm}$ size which will cap the $\mathrm{Al}$ islands and be dispersed in between. The Au clusters are then electrically and mechanically linked with each other and the read/write lines by molecular wire (organic or organo-metallic) and dendritic conducting polymers (Hideo et al 1988) using the 'molecular alligator clip' technology (Schumm et al 1996). For example, one can use thiolated polythiophenes for connecting Au clusters. Note that this also connects up the Al islands. Any diagonal connection formed between the $\mathrm{Al}$ islands in the array does not pose any problem; it simply provides an alternate path for mutual charging of the Al dots. Note that in principle, any molecule connected between dissimilar metals will rectify because the chemical potentials in dissimilar metals are different resulting in bending of the HOMO/LUMO levels. Thus it is relatively easy to obtain molecular rectification (diodes).

This memory element is a new design and is tailormade for self-assembly. It results in ultra-low power, ultrafast and ultradense electronic data storage. We can estimate the order of the speed and power figures by assuming a realistic value of $1 \mathrm{aF}$ for the dot capacitance $\left(C_{\mathrm{dot}}\right)$ and the molecular capacitance $\left(C_{\text {molecule }}\right.$ ) (Janes et al 1995). The switching delay is the RC time constant of the interconnect network $\left(=R_{\text {molecule }}\left(C_{\mathrm{dot}}+C_{\text {molecule }}\right)\right)$ which is $\sim 1$ picosecond if we assume the molecular resistance to be $1 \mathrm{M} \Omega$ (Janes et al 1995). The power-delay product is $\left(C_{\mathrm{dot}}+C_{\text {molecule }}\right) V^{2}$, where $V$ is the voltage swing for charging. Assuming $V=q / C_{\mathrm{dor}}$ (we can charge a dot with a single electron), the power delay product is $5 \times 10^{-20} \mathrm{~J}$ and hence the dynamic power dissipation for writing a bit is on the order of 50 nanowatts. These are excellent figures.

\subsection{Non-linear optical elements}

Quantum dots self-assembled by the second process of anodization have shown remarkable non-linear optical properties. We have prepared arrays of CdS quantum dots which have been studied by our collaborators with degenerate pump-probe spectroscopy.

Measurements of second harmonic signal accruing from optical excitation of $\mathrm{CdS}$ dots at a sub-bandgap frequency have shown that these structures can act as effective frequency converters with low insertion loss (Balandin et al 1997). Since the excitation energy in these experiments is well below the bandgap, we expect that the second-harmonic generation is associated with either giant dipole transitions between the quantized subbands in the conduction band (Svizhenko et al 1997) or multi-photon excitations (Sheik-Bahae et al 1990, 1991). The measured second-order non-linear dielectric susceptibility $\chi^{(2)}$ is five times larger than the value in bulk CdS (Balandin et al 1997) showing that quantum confinement has a pronounced effect on optical non-linearity. Such strong non-linearities make these structures very attractive for optical switching networks, mixers, limiters, optical logic gates and generic media for studying optical solitons.

\subsection{High density magnetic storage media}

Research in electrodeposition of ferromagnetic metals and alloys in nanoporous alumina films date back to the 1970s (Kawai and Veda 1975). These films exhibit magnetic anisotropy with the easy direction of magnetization perpendicular to the film plane. Also, large perpendicular coercivity and high magnetic remanence 
ratios are obtained. Such features make films of this type suitable for magnetic recording applications, especially for media leading to extremely high recording density, well in excess of 10 gigabits/inch ${ }^{2}$.

We have been investigating properties of cobalt deposited in nanopores. The nanoporous alumina film is formed by anodizing $99.99 \%$ pure $\mathrm{Al}$ foil in $15 \%$ sulfuric acid with a dc current density of $20 \mathrm{~mA} / \mathrm{cm}^{2}$ at room temperature for $10-45 \mathrm{~min}$. A minimum of $10 \mathrm{~min}$ of anodization is required for a well developed pore structure. The pores are then 'rounded' by soaking in sulfuric acid.

For electrodeposition of cobalt into the nanopores, a $0.1 \mathrm{M} \mathrm{CoSO}_{4}$ solution is used as an electrolyte. Various parameters are found to influence the magnetic properties of the resulting film. AC electrodeposition with a sine wave of frequency $100-200 \mathrm{~Hz}$ and amplitude $15-20 \mathrm{~V}$ rms, and a deposition time of about $30 \mathrm{sec}-2 \mathrm{~min}$ give the best results. The coercivity increases with time of deposition and tends to saturate past $2 \mathrm{~min}$.

In collaboration with others, we have measured extremely high magnetic coercivity of $2.17 \mathrm{kOe}$ and a remanence ratio of 0.75 in cobalt quantum dots (Bandyopadhyay et al 1998). The coercivity is larger than what has been reported before (Moskovitz and Schmid-Halter 1988; Zangari and Lambeth 1997). Details of these measurements and results will be reported elsewhere.

Electrodeposited Co in nanopores is a rich system for studying the correlation between nanostructure and magnetic properties. The interaction between magnetic grains and the magnetization reversal mechanism can be studied systematically by varying the particle size and inter-particle distance. Unlike conventional recording media in which the magnetic grains are physically contiguous, and the main interaction between the grains is exhange interaction, in this system, the magnetic grains are well-separated and the main interaction is magnetostatic. Additionally, the distribution in particle volume can be very narrow. This is extremely important in producing thermally stable media at ultra-high density (Sellmyer et al 1998). Finally, this system offers considerable versatility in the choice of materials. Both metals and alloys, as well as layered structures, can be electrodeposited into the pores to yield a variety of magnetic materials with very high coercivity required for high density recording.

\subsection{Mesoscopic superconductors}

High $T_{\mathrm{c}}$ superconductor YBCO was electrodeposited in alumina nanopores with a view to producing mesoscopic superconducting quantum dots. High $T_{\mathrm{c}}$ materials are suitable for this purpose since the coherence length of Cooper pairs in bulk materials can be quite small $(<1 \mathrm{~nm})$ so that the pairs are tightly bound and the pairing can occur coherently within a quantum dot whose diameter is less than $10 \mathrm{~nm}$. These structures can theoretically exhibit increased transition temperature since the density of states at the Fermi level can be very large if the Fermi level coincides with a subband edge.

Our initial experiments have shown a $T_{\mathrm{c}}$ of only $88 \mathrm{~K}$ which is somewhat less than what is found in bulk materials. It is quite possible that impurity broadening of the subband levels significantly reduces the density of states and thus fails to increase the $T_{c}$. We are currently attempting to carry out 'density of states engineering' by attaching a back gate to the substrate in an effort to manipulate the Fermi level alignment with a subband edge. This appears to be a promising approach. Very recently, we have succeeded in increasing the $T_{\mathrm{c}}$ to $96 \mathrm{~K}$ in $\mathrm{YBCO}$ dots.

\section{Conclusion}

In conclusion, we have described some recent results pertaining to the topographical, optical, electronic, superconducting and magnetic properties of electrochemically self-ordered quantum dot arrays. If successful, these endeavors can lead to useful optical, electronic, superconducting and magnetic devices, as well as novel materials such as photonic crystals, etc.

\section{Acknowledgements}

The work on self-assembly has been performed in collaboration with Prof. A E Miller, Prof. H C Chang, Dr G Banerjee, and Dr D-F Yue, Department of Chemical Engineering, University of Notre Dame, USA. The work on non-linear optical properties of CdS quantum dots was performed in collaboration with D Zarestsky and co-workers in the Kurchatov Institute, Moscow, Russia. Much of the initial optical characterization was performed by Prof. Meera Chandrasekhar, University of MissouriColumbia, USA. Magnetic measurements were carried out by Prof. David Sellmyer, Dr Latika Menon and Mr H Zeng, University of Nebraska, USA. Topographical studies are mostly the work of Mr Nikolai Kouklin at Nebraska. Measurements of the transition temperature of superconductors were performed by Prof. Howard Blackstead, University of Notre Dame, USA.

Various components of this work were supported by the US Army Research Office under grant DAAG55-981-0015 and by the Defense Advanced Research Projects Agency under ULTRA contract 35918-OH. Additional support was received from the Centre for Materials Research and Analysis founded under the Nebraska Research Initiative and from the Midwest Superconductivity Consortium through a US Department of Energy grant DE-FG02-90ER45427. 
The author is grateful for the hospitality of the Indian Institute of Science, Bangalore during the IUMRS-98 conference.

\section{References}

Andres R P, Bielefeld J D, Henderson J I, Janes D B, Kolagunta V R, Kubiak C P, Mahoney W J and Osifchin R G, 1996 Science 2731690

Balandin A, Bandyopadhyay S, Snyder P G, Stefanovich S, Varfolomeev A, Zaretsky D, Banerjee $G$ and Miller A E, 1997 Phys. Low Dim. Struct. 11/12 155

Bandyopadhyay S, Miller A E, Chang H-C, Banerjee G, Yuzhakov V, Yue D-F, Ricker R E, Jones S, Eastman J A, Baugher E and Chandrasekhar M 1996 Nanotechnology 7360

Bandyopadhyay S, Menon L, Kouklin N, Zeng H and Sellmyer D J 1998 J. Elec. Mat. (submitted)

Bimberg D, Grundmann M and Ledentsov S 1998 Quantum dot heterostructures (New York: John Wiley \& Sons)

Diggle J W, Downie T C and Goulding C W 1969 Chem. Rev. 69365

Hideo $\mathrm{T}$ et al $1988 \mathrm{~J}$. Am. Chem. Soc. 1204492

Huber C A, Huber T E, Mqio Sad, Lubin J A, Manalis S and Prater C B 1994 Science 263800

Janes D B, Kolagunta V R, Osifchin R G, Bielefeld J D, Andres R P, Henderson J I and Kubiak C P 1995 Superlat \& Microstruct. 18275

Kawai S and Ueda R 1975 J. Electrochem. Soc. 12132

$\mathrm{Li}$ Fei Yue 1997 Nanostructure of anodic porous alumina films of interest in magnetic recording, $\mathrm{Ph} d$ Dissertation, University of Alabama, Huntsville
Martin C R 1994 Science 2661961

Masuda H, Yamada H, Satoh M, Asoh H, Nakao $M$ and Tamamura T 1997 Appl. Phys. Lett. 712770

Miller A E and Bandyopadhyay S, 1998 US Patent \#5,747, 180 Moskovitz M 1993 US Patent \#5,202,290

Moskovitz M and Schmid-Halter B 1988 International Patent publication \#WO88/02538

Murray C B, Kagan C R and Bawendi M G 1995 Science 270 1335

Ricker R E, Miller A E, Yue D-F, Banerjee G and Bandyopadhyay S 1996 J. Elec. Mat. 251585

Routkevich D, Tager A A, Haruyama J, Almawlawi D, Moskovitz M and Xu J M 1996 IEEE Trans. Elec. Dev. 431646

Roychowdhury V P, Janes D B, Bandyopadhyay S and Wang X 1996 IEEE Trans. Electron Dev. 431688

Roychowdhury V P, Janes D B and Bandyopadhyay S 1997 Proc. IEEE 85574

Schumm J S, Pearson D L, Jones L II, Hara R and Tour J M 1996 Nanotechnology 7430

Sellmyer D J, Yu M, Thomas R A, Liu Y and Kirby R D 1998 Phys. Low Dim. Struct. 1/2 155

Sheik-Bahae M et al 1990 Phys. Rev. Lett. 6596

Sheik-Bahae M et al 1991 IEEE J. Quantum Electron. 27 1296

Svizhenko A, Balandin A and Bandyopadhyay S 1997 J. Appl. Phys. 817927

Tada T et al 1998 J. Phys. D31 L21

Yue D-F, Banerjee G, Miller A E and Bandyopadhyay S 1996 Superlat. Microstruct. 19191

Yuzhakov Vadim V, Chang Hsueh-Chia and Miller Albert E 1997 Phys. Rev. B56 12608

Zangari G and Lambeth D N 1997 IEEE Trans. Magn. 333010 